\title{
Corrigendum
}

\section{Corrigendum to "Anticancer Effects of Salvia miltiorrhiza Alcohol Extract on Oral Squamous Carcinoma Cells"}

\author{
Wen-Hung Wang, ${ }^{1,2,3}$ Yu-Hsuan Kuo, ${ }^{4}$ Ling-Ya Chu, ${ }^{5}$ Chia-Ying Lee, ${ }^{5}$ \\ Yu-Chang Tyan, ${ }^{6,7,8,9,10}$ Zong-Shiow Chen, ${ }^{11}$ and Wan-Chi Tsai ${ }^{5,7}$ \\ ${ }^{1}$ Department of Otolaryngology, Cathay General Hospital, Taipei City 106, Taiwan \\ ${ }^{2}$ Department of Otolaryngology, Sijhih Cathay General Hospital, New Taipei City 221, Taiwan \\ ${ }^{3}$ School of Medicine, Fu-Jen Catholic University, New Taipei City 242, Taiwan \\ ${ }^{4}$ Department of Hemato-Oncology, Chi-Mei Medical Center, Tainan City 710, Taiwan \\ ${ }^{5}$ Department of Medical Laboratory Science and Biotechnology, Kaohsiung Medical University, Kaohsiung 807, Taiwan \\ ${ }^{6}$ Department of Medical Imaging and Radiological Sciences, Kaohsiung Medical University, Kaohsiung 807, Taiwan \\ ${ }^{7}$ Center for Infectious Disease and Cancer Research, Kaohsiung Medical University, Kaohsiung 807, Taiwan \\ ${ }^{8}$ Graduate Institute of Medicine, College of Medicine, Kaohsiung Medical University, Kaohsiung 807, Taiwan \\ ${ }^{9}$ Institute of Medical Science and Technology, National Sun Yat-Sen University, Kaohsiung 804, Taiwan \\ ${ }^{10}$ Department of Medical Research, Kaohsiung Medical University Hospital, Kaohsiung 807, Taiwan \\ ${ }^{11}$ Institute of Cosmetic Science, Chia-Nan University of Pharmacy and Science, Tainan City 717, Taiwan
}

Correspondence should be addressed to Wan-Chi Tsai; wanchi@kmu.edu.tw

Received 2 April 2017; Accepted 6 April 2017; Published 11 May 2017

Copyright (C) 2017 Wen-Hung Wang et al. This is an open access article distributed under the Creative Commons Attribution License, which permits unrestricted use, distribution, and reproduction in any medium, provided the original work is properly cited.

In the article titled "Anticancer Effects of Salvia miltiorrhiza Alcohol Extract on Oral Squamous Carcinoma Cells" [1], the name of the second author was given incorrectly as Kuo-Yu Hsuan. The author's name should have been written as YuHsuan Kuo. The revised authors' list is shown above.

\section{References}

[1] W.-H. Wang, K.-Y. Hsuan, L.-Y. Chu et al., "Anticancer effects of Salvia miltiorrhiza alcohol extract on oral squamous carcinoma cells," Evidence-Based Complementary and Alternative Medicine, vol. 2017, Article ID 5364010, 9 pages, 2017. 


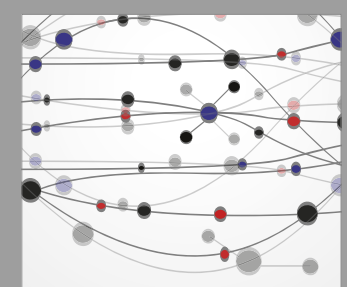

The Scientific World Journal
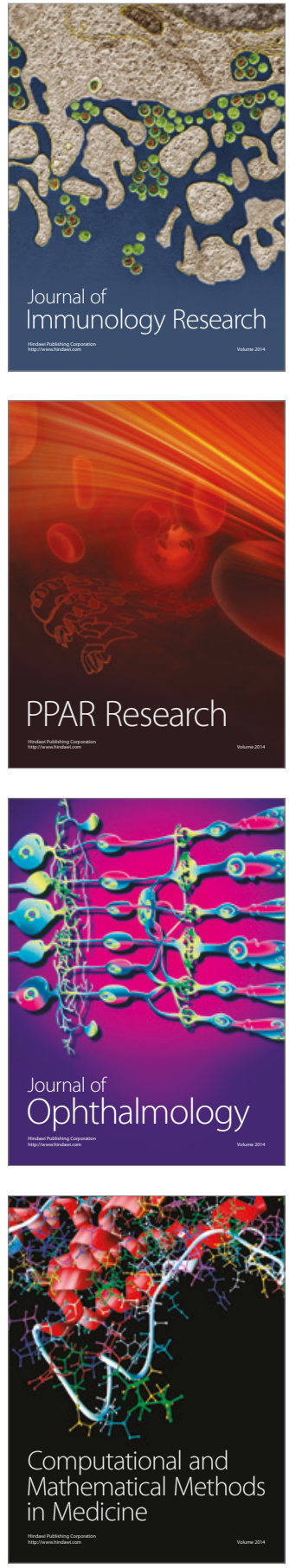

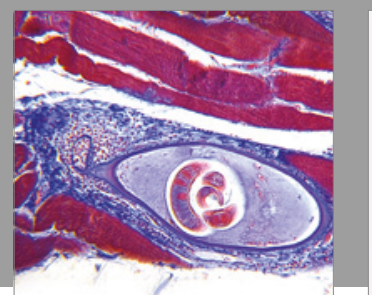

Gastroenterology Research and Practice
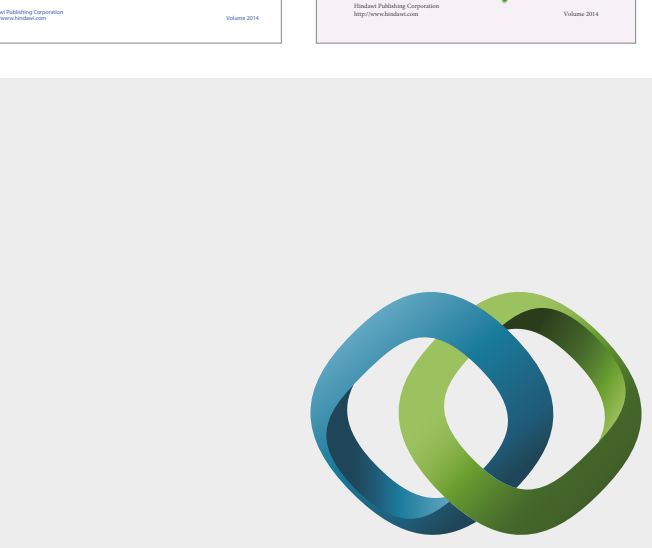

\section{Hindawi}

Submit your manuscripts at

https://www.hindawi.com
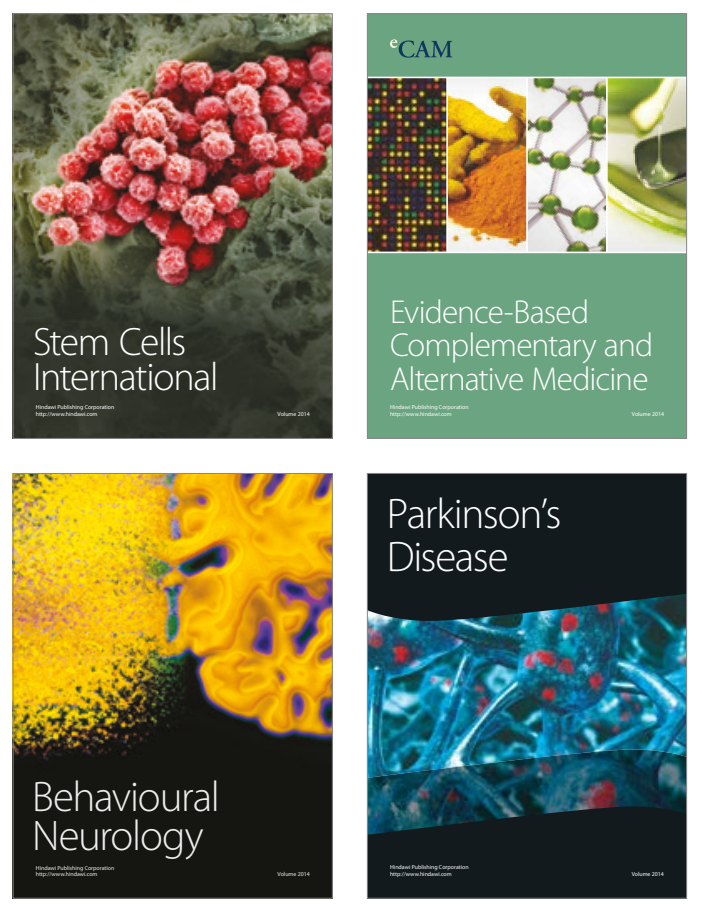
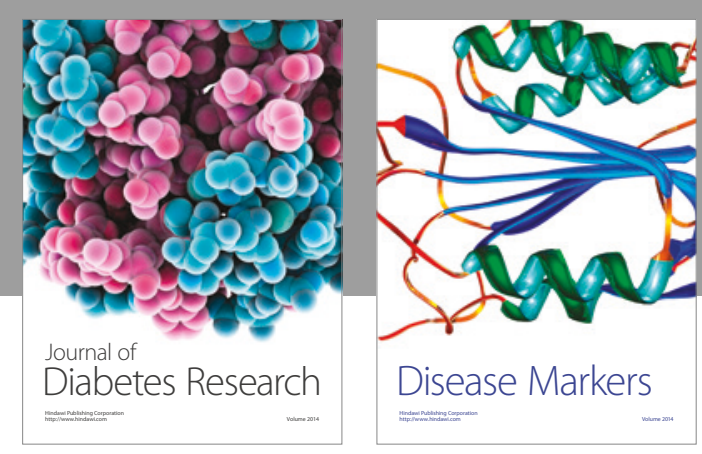

Disease Markers
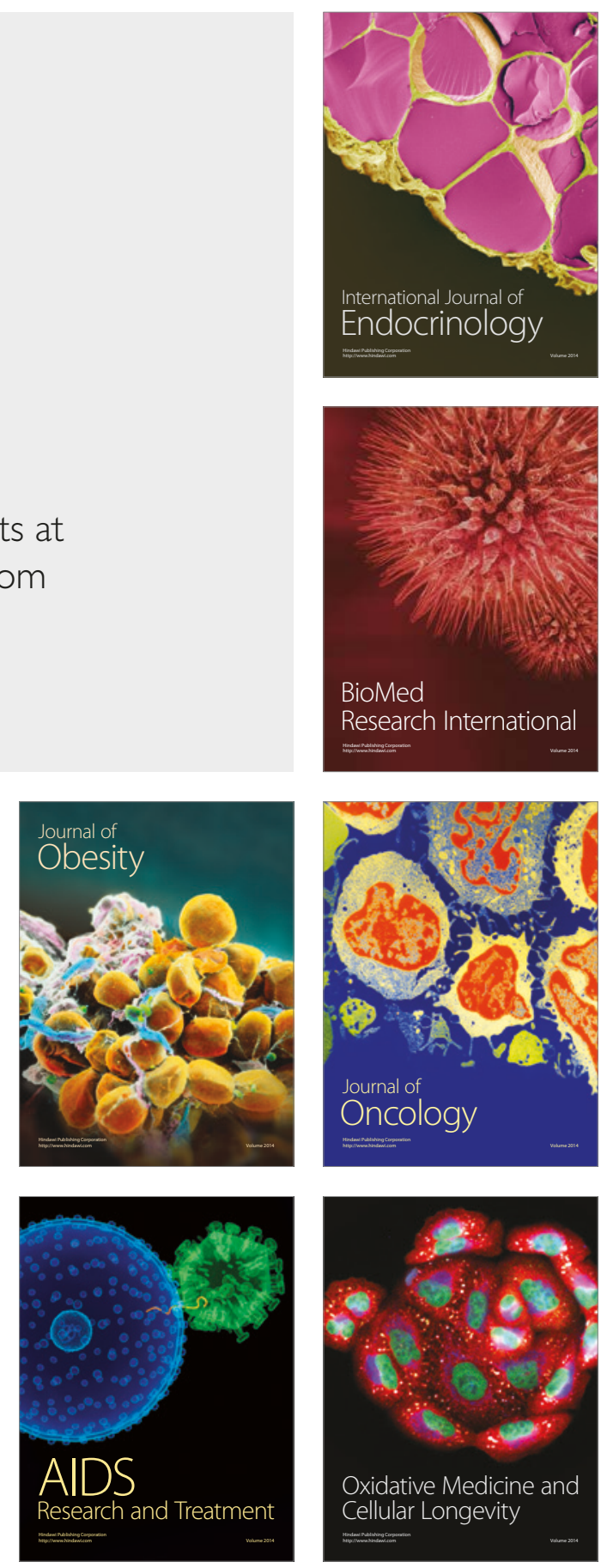